\title{
A grid refinement approach for a three-dimensional soil-root water transfer model
}

\author{
T. Schröder, ${ }^{1}$ L. Tang, ${ }^{2}$ M. Javaux, ${ }^{3,4}$ J. Vanderborght, ${ }^{3}$ B. Körfgen, ${ }^{1}$ and H. Vereecken ${ }^{3}$ \\ Received 17 February 2009; revised 9 July 2009; accepted 29 July 2009; published 8 October 2009.
}

[1] Three dimensional soil-root water transfer models require a fine soil and root discretization in order to obtain accurate results. This goes along with a considerable computational effort. One way of reducing the computational effort is the usage of grid refinement techniques. With such techniques irregular grids are obtained that combine the accuracy of a fine grid resolution with a considerable reduction in computational costs. As a consequence of plant transpiration roots take up water and large soil water potential gradients around roots are created. Especially in these regions a fine soil discretization is needed. The root spatial distribution can therefore be used for refinement of the soil grid, a priori. Simulations show that the accuracy is indeed maintained for a priori refined grids but with reduced computational costs as compared to regular fine grids. Comparison with a well recognized a posteriori error estimate strengthen these results.

Citation: Schröder, T., L. Tang, M. Javaux, J. Vanderborght, B. Körfgen, and H. Vereecken (2009), A grid refinement approach for a three-dimensional soil-root water transfer model, Water Resour. Res., 45, W10412, doi:10.1029/2009WR007873.

\section{Introduction}

[2] For a variety of environmental and agricultural purposes, such as irrigation management or yield prediction under water scarcity conditions, the understanding of root water uptake processes is of importance. Model development have lead to three-dimensional soil-root water flow models that describe the relationship between water flow in soil and the uptake of water by plant roots [Javaux et al., 2008]. In such models the root structure is independently coupled to the soil grid that is used for the numerical solution of the soil water flow equation, i.e., the Richards equation. Because of more accurate measurement techniques [Pohlmeier et al., 2008] and improved root growth and root architecture models [Pagès et al., 2004] more detailed root architectures can be obtained. Furthermore, to acquire high resolution soil water potentials and velocity profiles (for solute transport) a fine soil discretization is needed [Schröder et al., 2009]. As a consequence, computational costs increase largely for estimation of the water potentials in the soil domain enclosing detailed root structures. Grid refinement techniques can be used to reduce computational costs by increasing the spatial resolution at locations where high gradients in soil water potential and pore water velocity exist, and using a coarser discretization at other locations [Mansell et al., 2002]. This effectively decreases the number of elements used in the numerical grid but at the same time

\footnotetext{
${ }^{1}$ Jülich Supercomputing Center, Forschungszentrum Jülich GmbH, Juelich, Germany.

${ }^{2}$ Department of Applied Mathematics, University of Colorado, Boulder, Colorado, USA.

${ }^{3}$ Institute for Chemistry and Dynamics of the Geosphere, Agrosphere Institute, Forschungszentrum Jülich, Juelich, Germany.

${ }^{4}$ Department of Environmental Sciences and Land Use Planning, Université Catholique de Louvain, Louvain-la-Neuve, Belgium.

Copyright 2009 by the American Geophysical Union. 0043-1397/09/2009WR007873
}

keeps the high spatial resolution in parts of the domain where gradients are large.

[3] A generally used method for grid refinement is the a posteriori error estimate [Babuška and Rheinboldt, 1978]. In this method the error between exact and approximated solution for the elements of the soil grid is calculated after the linear system of equations is solved. If the error exceeds a certain threshold value the element is refined. The a posteriori error estimate is a dynamic approach where the soil resolution increases (or decreases) over time. Dynamic approaches could, e.g., be used for modeling root growth. For two-dimensional soil grids with a two-dimensional root growing system Wilderotter [2003] imposed such a scheme. A second method for grid refinement is the usage of a priori information. In root water uptake models, it is expected that, due to transpiration, large soil water potential gradients will occur around active roots. Therefore a finer soil discretization in the vicinity of roots is required [see, e.g., Schröder et al., 2008]. An a priori grid can be developed based on the root architecture with finer elements near roots and coarser elements further away. A priori refinement can either be used in a static or dynamic way. A static grid is defined as a grid that does not change over time and is acquired after considering the total root structure. A dynamic grid is only based on that part of the root structure that is active and takes up water, and is time dependent.

[4] The problems that arise with refinement techniques for coupled three-dimensional soil-root water flow models are related to the size of the soil discretization. First, the Richards equation is only valid for a soil discretization larger than the representative elementary volume of the soil texture [Bear, 1972]. Second, if very small soil elements are used the volume of soil around a root is very small, such that the ratio of the uptake rate to the volume of soil (i.e., the sink term) becomes very large causing the system of equations to diverge. Therefore a theoretical and methodological limit exists in the minimum dimension of a soil element. 
[5] In this paper we developed a grid refinement approach based on a priori root information that can be used in either a static or dynamic way. We first compared a regular fine grid (reference grid) with three regular coarser grids and three static refined grids based on a priori root information in terms of accuracy and computational time for a given soil-root scenario. Furthermore, the obtained static grids using a priori information are compared and validated with grids obtained by an a posteriori error estimate, that was deduced for the three-dimensional water flow equation. In order to compare both methods the a posteriori refinement method is adapted to generate "static" grids. Finally, we address the usage of dynamic a priori refinement techniques for the presented three-dimensional soil-root water flow model.

\section{Methods}

\subsection{Three-Dimensional Macroscopic Water Flow Model in Soil and Roots}

[6] The model of Javaux et al. [2008] consists of two interacting systems: the soil matrix and the root architecture. The soil system is discretized into cubes that are again divided into six linear tetrahedral elements of equal shape, to be used for numerical interpolation of the Richards equation. The root system is divided into nodes connected by segments. For both systems a set of equations is solved in terms of water potential and both are coupled via the sink term $S$ in the Richards equation, see the work of Javaux et al. [2008] for more details. The boundary conditions for the plant root system are a transpiration rate or a water potential at the root collar, and a soil water potential at the soil-root interface enabling calculation of the water potential within the roots. Stress is defined when the water potential at the root collar is lower than a limiting water potential value. From this point on the water potential is kept constant at the root collar so that the simulated actual transpiration rate becomes smaller than the potential transpiration rate.

[7] Radial soil-root water flow $J_{r}\left[\mathrm{~cm}^{3} \mathrm{~d}^{-1}\right]$ is given by

$$
J_{r}=L_{r}^{*} A_{r}\left(\psi_{\text {int }}-\psi_{\text {xylem }}\right)
$$

where $L_{r}^{*}$ is the radial root conductivity $\left[\mathrm{d}^{-1}\right], A_{r}\left[\mathrm{~cm}^{2}\right]$ the root outer surface and $\psi[\mathrm{cm}]$ represents the water potential expressed as hydraulic head at the soil-root interface $\left(\psi_{\text {int }}\right)$ and in the xylem $\left(\psi_{x y l e m}\right)$ respectively. The outer root surface is defined by $A_{r}=2 \pi r_{i n t} l_{r}$, with $r_{i n t}[\mathrm{~cm}]$ the root radius and $l_{r}[\mathrm{~cm}]$ the length of the root segment. The water potential at the soil-root interface is estimated by a distancebased weighting function of the water potential in the surrounding soil nodes. The sink term $\left[\mathrm{d}^{-1}\right]$ of a soil cube $j$ is calculated by

$$
S_{j}=\frac{\sum_{k=1}^{n_{j}} J_{r, k}}{V_{j}}
$$

where the nominator represents the sum of all the radial soil-root fluxes of the $n_{j}$ root nodes located inside a soil cube $j$ and $V_{j}\left[\mathrm{~cm}^{3}\right]$ is the volume of the $j$ th soil cube. A root node is defined as the center of a root segment at which water exchange is allowed. The sink term is then distributed upon the soil nodes $i$ representing the bulk soil

$$
S_{i}=S_{j} w_{i} \frac{\frac{\left(\psi_{i}-\psi_{i t}\right)}{d i s t_{i}}}{\sum_{l=1}^{8} \frac{\left(\psi_{l}-\psi_{\text {int }}\right)}{\text { dist }_{l}}}, \quad \text { for } i=1, \ldots, 8
$$

where dist is the distance from the soil node $i$ to the soilroot interface and $w_{i}$ is a volume fraction that relates the volume of soil belonging to soil node $i$ with the volume of the soil cube $V_{j}$.

[8] For each timestep of the simulation first the root system is solved, after that the soil system and again the root system in an iterative way. If the changes in both systems are lower than imposed tolerance criteria, for water content and water potential regarding the soil system and for xylem water potential regarding the root system, the final solution for this timestep is found and the simulation continues with the next timestep.

\subsection{A Priori Refinement}

[9] The initial, regular coarse grid is divided into cubes which are again divided into elements. The a priori refinement technique is applied to the soil cubes not the elements. Firstly, to enable the usage of equation (2). Secondly, because of lower computational costs of routines that are needed for equations (1) and (2) when using cubes instead of elements. As the discretization size of a cube is limited a multilevel refinement technique up to a minimal grid size is employed. This technique is based on bisections of the initial coarse grid, without additional coarsening. A twolevel refinement scheme is demonstrated in Figure 1 using the known root information. Consider a root node positioned randomly in a soil cube as given in Figure 1a. Then we (1) bisect this cube in eight parts (Figure 1b), (2) identify the subcube containing the root node, (3) bisect the identified cube in additional eight cubes (Figure 1c).

[10] For a static grid this procedure is performed for all root nodes in the given soil domain, for a dynamic grid only a selection of root nodes is considered. After this procedure a first refined grid based on the a priori root information is gained.

[11] Due to the multilevel refinement smaller soil cubes were generated with so-called slave nodes. Slave nodes are soil nodes that are not connected to another soil node in all available directions of the three-dimensional grid. Master nodes, on the other hand, are connected in all possible directions, i.e., in 6 directions if the soil node is not located at the soil boundary domain. Slave nodes are located on the six faces of a coarser soil cube bounding its subcubes (crosses in Figure 1c). Two problems occur when using the multilevel refinement technique. The first problem is related to the allocation of a sink term to a soil node in the threedimensional model. In the case of regular grids (all master nodes) each soil node has a predefined volume of soil including nodes located at the soil boundary domain. This volume of soil is used in equation (2) to estimate the sink term obtained from the radial water flow of the root system. Allocation of a soil volume to a slave node is rather complicated, as the volume of soil is not necessarily equal to the volume of the cubes the slave node belongs to. An example is illustrated in Figure 1c. A slave node belongs in 


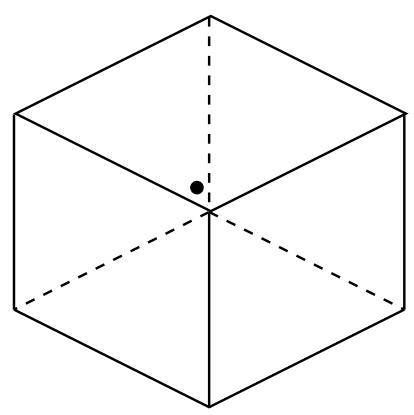

A

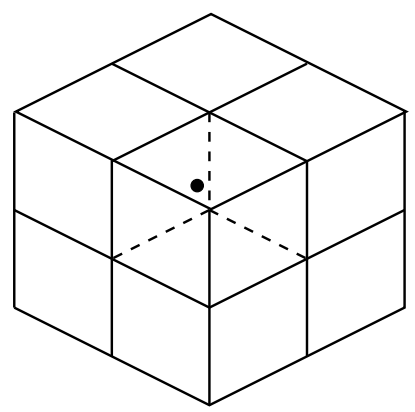

B

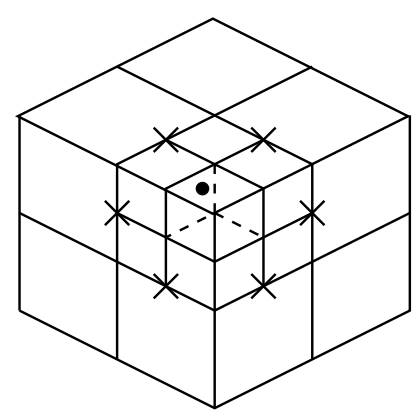

$\mathrm{C}$

Figure 1. Bisection of a soil cube. (a) A coarse cube with a root node (black dot). This cube is split in equal parts and (b) the refined cube is obtained. This cube is divided again in the subcube containing the root node and leads to (c) the refined grid. In Figure 1c slave nodes (crosses) are denoted, but only those at the drawn outer soil surfaces.

this example to two fine soil cubes, however, it has also influence on the larger adjacent cube without being an actual node of this cube. The grid is therefore extended around the initial multilevel refined grid to transform a slave node, that has been allocated a sink term, into a master node. Hereto neighboring cubes, adjacent to the cube with the slave node, with a refinement level larger than the minimal refinement level are refined to this minimal level. The second problem deals with the derivative of the solution variable (water potential) at the interface between coarser and finer cubes. After the initial multilevel refinement only soil cubes with root nodes in it are refined. It may very well occur that an unrefined coarser soil cube is located next to a refined coarser cube (where the difference in level of refinement is two or higher). In this case large jumps in the derivative of the solution variable are expected. To predict a smooth gradient throughout the soil the transition from coarser cubes to finer cubes and vice versa is restricted to a stepsize of one in the level of refinement.

\subsection{Dynamic A Priori Refinement Based on Active Root Segments}

[12] A static a priori grid is gained if all root nodes are considered for refinement of the soil grid. It would be ideal for assuming that all root nodes take up water simultaneously. This is not the case in reality though, only part of the root system is active [Passioura, 1980] as is shown by simulations of Javaux et al. [2008] and Schröder et al. [2009]. Thus the disadvantage of the static grid is that the number of soil nodes is generally overestimated. Furthermore, the root system may grow and finer soil cubes may be required. Therefore a dynamic approach is introduced. Initially the simulation is started with a coarse grid. After the root system is solved, only those root nodes with a radial soil-root water flow are considered and refinement is performed as in the previous section. Furthermore, a criterion is introduced such that refinement is performed again if this criterion is exceeded. For each timestep the difference in radial soil-root water flow between the current timestep and the one on which previous refinement was considered, is calculated. However, only for those root nodes around which the soil is not yet refined. If the sum of the absolute differences is larger than $5 \%$ of the total absolute sum of the radial soil-root water fluxes (at the current timestep) then refinement is allowed. Refinement can be performed in addition to the already existing grid. However, root water uptake patterns may shift largely between soil layers [ $\mathrm{Li}$ et al., 2002; Schröder et al., 2008] and the refined grid may have soil cubes that equal the minimum discretization size but where no refinement is actually desired, as the roots do not take up water anymore. Therefore the initial coarse grid is taken as point of departure for each refinement and refinement is performed for only those soil cubes that have root nodes with a radial soil-root flow unequal to zero.

\subsection{A Posteriori Refinement}

[13] The a posteriori error estimate is a widely used dynamic method for grid refinement in soil water flow models [Mansell et al., 2002]. Based on the a posteriori error estimate a grid that produces the smallest error between the approximated and exact solutions (theory describing the approximated and exact solution is given in detail in [e.g., Briggs et al., 2000]) for the current time step can be created. The major disadvantage is that the obtained grid is not fulfilling the criteria that were posed for the three-dimensional soil-root model, namely the slave nodes should not have a denoted sink term and the transitions in grid size between two adjacent soil cubes should not exceed a stepsize of one.

[14] Here the a posteriori error estimate is used as a tool to validate the a priori refinement method. A derivation of the a posteriori error estimate is given in Appendix A and a short description is given hereafter. After the system of equations is solved with some method the error of an element $T$ can be determined. The error depends on the residual values of the vertices of an element (element residual) and on the residual values of the faces of an element (jump residual). From these residuals the so-called local error indicator $\left(\eta_{H}\right)$ of an element can be deduced. Another important concept, oscillation $\left(o s c_{H}\right)$, accounts for information missed by the averaging process associated with the employed finite element method. The local error indicator and the oscillation are important parameters for a posteriori refinement. If refinement is required, by evaluation of a threshold value, then elements can be marked based on these two parameters. The first marking strategy selects the minimal subset of elements $\hat{T}_{H}$ of the mesh $\mathcal{T}_{H}$ such that

$$
\sum_{T \in \hat{\mathcal{T}}_{H}} \eta_{H}(T)^{2} \geq \theta_{E}^{2} \eta_{H}(\Omega)^{2}
$$


Table 1. Grid Configurations That Are Evaluated for the SoilRoot Scenario

\begin{tabular}{cll}
\hline Scenario & Type of Refinement & Initial Soil Discretization $(\mathrm{cm})$ \\
\hline 1 & 0-level refinement & 0.25 by 0.25 by 0.25 (reference) \\
2 & 0-level refinement & 0.5 by 0.5 by 0.5 \\
3 & 0-level refinement & 1 by 1 by 1 \\
4 & 0-level refinement & 2 by 2 by 2 \\
5 & 1-level refinement & 0.5 by 0.5 by 0.5 \\
6 & 2-level refinement & 1 by 1 by 1 \\
7 & 3-level refinement & 2 by 2 by 2 \\
\hline
\end{tabular}

with $0<\theta_{\mathrm{E}}<1$ and $\Omega$ consists of all elements. The second strategy replaces in equation (4) the error with oscillation

$$
\sum_{T \in \hat{\mathcal{T}}_{H}} \operatorname{osc}_{H}(T)^{2} \geq \theta_{o}^{2} O s c_{H}(\Omega)^{2}
$$

with $0<\theta_{\mathrm{o}}<1$. The parameters $\theta_{\mathrm{E}, o}$ are threshold parameters that determine the number of selected elements that fulfill equations (4) and (5). Note that, equivalent to the multilevel refinement strategy, the soil cubes with the marked elements are refined.

\subsection{Simulation Scenarios}

[15] A soil-root scenario is defined to demonstrate the refinement techniques discussed in this paper. The soil-root scenario consists of a soil column with dimensions 10 by 10 by $34 \mathrm{~cm}$. The texture is a loamy soil with parameters $\theta_{r}=$ $0.08 \mathrm{~cm}^{3} \mathrm{~cm}^{-3}, \theta_{s}=0.43 \mathrm{~cm}^{3} \mathrm{~cm}^{-3}, K_{s}=50 \mathrm{~cm} \mathrm{~d}^{-1}, \alpha=$ $0.04 \mathrm{~cm}^{-1}, \lambda=0.5$ and $\mathrm{n}=1.6$ [Carsel and Parrish, 1988]. The Mualem-van Genuchten parameterization [van Genuchten, 1980 ] is used to evaluate the soil characteristics $K(\psi)$ and $\theta$ needed by the Richards equation. The soil is considered homogeneous with an initial water potential of $-300 \mathrm{~cm}$ throughout the soil. The root structure is a $500 \mathrm{~h}$ old root generated by the model of Somma et al. [1998] and consists of 9488 root segments. Root system hydraulic parameters dependent on root segment age, for radial as well as axial flow within the roots, were taken from the work of Doussan et al. [1998]. Only water flow, neither solute transport nor root growth is simulated. Zero fluxes at the top, bottom and lateral boundaries of the soil domain are imposed. Two type of root collar boundary conditions are used for the simulations. First, a constant water potential at the root collar equal to $-15,000 \mathrm{~cm}$ is imposed. Simulations are performed over 5 days and the amount of water taken up by the roots, expressed in the actual transpiration rate, is evaluated. Second, a constant flux at the root collar equal to $15 \mathrm{~cm}^{3} \mathrm{~d}^{-1}$ is imposed. The transpiration of water by the plant causes a continuous water uptake by the roots and the simulation is stopped if the xylem water potential reaches a certain water potential threshold value at the root collar $(-15,000 \mathrm{~cm})$, thereby simulating water stress conditions. This boundary condition allows for investigation of soil-root interactions in locally dry soil regions that will develop around roots [Schröder et al., 2009].

\subsubsection{Comparison of Static Irregular A Priori Grids With Regular Grids}

[16] Several scenarios were simulated to compare the performances of the model with irregular versus regular soil grids. Table 1 summarizes the 7 scenarios. Scenarios $1-4$ are the standard nonrefined cases (0-level refinement) with different levels of discretization. Cases 5-7 represent scenarios in which static a priori refined grids were generated: based on the total root architecture and which do not change over time. These cases differ by their initial soil discretization and their level of refinement. After refinement, grids are generated that end up with fine soil cubes $(0.25$ by 0.25 by $0.25 \mathrm{~cm}$ ) around roots, equal to the minimum soil discretization that was set for scenario 1; reference scenario. Both flux and water potential boundary conditions were used to compare scenario 2-7 with the reference grid configuration in terms of accuracy and computational time.

\subsubsection{A Priori Versus A Posteriori Refinement}

[17] The second comparison that is performed is between a static a priori grid (scenario 6, Table 1) and a posteriori grids. The a posteriori refinement method is adapted for this purpose. Root water uptake is imposed for all root segments of the root system, with a sink value equal to $1 \mathrm{e}-3 \mathrm{~d}^{-1}$. The objective here is to investigate whether the static grid obtained with the a priori refinement method is similar to the one obtained with the well known a posteriori error estimate. Generation of the grid by using an a posteriori error estimator starts with an initial coarse grid of 1 by 1 by $1 \mathrm{~cm}$ soil cubes and refinement is allowed up to a minimum discretization size $(0.25$ by 0.25 by $0.25 \mathrm{~cm})$. Marking procedures are alternated between local error (equation (4)) and oscillation (equation (5)) for 4 cases, see Table 2. With these cases the effect of the marking sequences can be evaluated. If a new grid is gained after refinement, the same simulation time is rerun until a grid is obtained where all soil cubes with a root segment, that takes up water, equal the minimum discretization size. This methodological criterion is similar as the one for static a priori refinement, and is used in order to get a fair comparison between the two refinement methods. However, based on mathematical rules equation (A11) should be evaluated for the a posteriori refinement technique. After the final grid is acquired it is expanded to ensure mass conservation (slave nodes with a denoted sink are extended to master nodes). From this moment on we let the model run similarly to the static a priori grid using the flux root collar boundary condition. It may seem trivial to compare these cases but these comparisons are performed mainly for validation purposes of the a priori grid. Secondly, information is gained on the accuracy of simulations with a posteriori grids, on the effect of the marking sequences and on the computational time to create a priori versus a posteriori grids.

\subsubsection{Dynamic A Priori Refinement Based on Active Root Segments}

[18] A third comparison evaluates dynamic a priori refinement based on active root segments. We consider a

Table 2. The A Posteriori Scenarios for Different Marking Sequence Parameters to be Used for a Two-Level Refinement Scheme for the Soil-Root Scenario With a Flux Type Root Collar Boundary Condition

\begin{tabular}{lccc}
\hline Scenario & Type of Refinement & $\theta_{\mathrm{E}}$ & $\theta_{\mathrm{o}}$ \\
\hline Post 1 & a posteriori & 0.6 & 0.4 \\
Post 2 & a posteriori & 0.6 & 0.3 \\
Post 3 & a posteriori & 0.5 & 0.5 \\
Post 4 & a posteriori & 0.5 & 0.2 \\
\hline
\end{tabular}




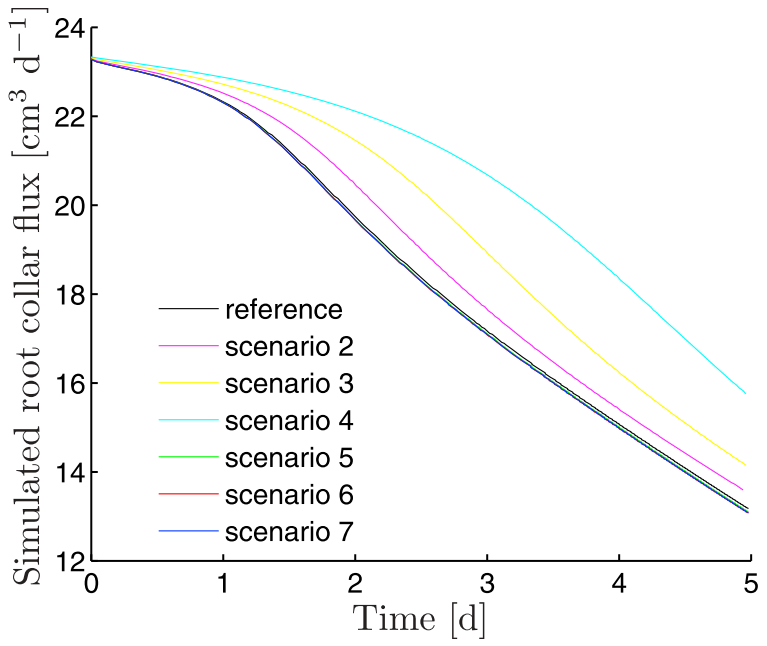

Figure 2. Simulated root collar fluxes for scenarios 1-7 of Table 1 with a water potential root collar boundary condition.

two-level refinement scheme (equivalent to scenario 6 of Table 1) and again we impose the flux boundary condition of $15 \mathrm{~cm}^{3} \mathrm{~d}^{-1}$ at the root collar. The results are compared in terms of accuracy and computational time with the reference case (scenario 1 of Table 1). Furthermore, they are compared with the results obtained using static a priori grids.

\section{Results and Discussion}

\subsection{Comparison of Static Irregular A Priori Grids With Regular Grids}

[19] First the scenario with the water potential boundary condition at the root collar is compared for the seven grid configurations of Table 1 . Such root collar boundary condition generates a decrease of the root collar flux over time since the water potential gradient between the root xylem tissue and the soil diminishes with soil water depletion. This is observed in Figure 2. The area under the curves equals the total amount of water that is taken up from the soil. Evaluating the coarse grid configurations (scenarios 2 (magenta), 3 (yellow) and 4 (cyan)) a less sharp decrease in simulated transpiration rate is noticed compared to the reference scenario (black line), meaning that more water is extracted from the soil. Thus following equation (1), the water potential estimate at the soil-root interface has a higher value than the reference scenario. This was expected from results shown by Schröder et al. [2008]. The a priori refined grids (scenarios 5 (green), 6 (red) and 7 (blue)) on the other hand are hardly distinctive with the reference case, indicating that the plant architecture indeed provides a very good estimate for the locations where high soil water potential gradients will occur.

[20] The accuracy of the solutions obtained with the different grid configurations can be tested over the whole water potential range using a flux boundary condition at the root collar. We are especially interested in dry soil regions that develop around roots over time. Soil water potential and xylem water potential distributions were compared at three simulation times $(0.5,4$ and $5.4 \mathrm{~d})$. The first simulation time $(0.5 \mathrm{~d})$ depicts wet soil conditions. At the second simulation time $(4 \mathrm{~d})$ the soil around the roots is much dryer and at the third simulation time $(5.4 \mathrm{~d})$ the limiting threshold value at the root collar is almost reached for the a priori refined scenarios and the reference case. From a soil point of view we compare the root mean square error (RMSE) of the soil water potentials of scenario 2-7 with the reference scenario at an arbitrary $x-y$ cross section $(\mathrm{z}=$ $-2 \mathrm{~cm}$ ) in the soil column. Note that the root length density is high in this cross-sectional plane (not shown). The RMSE values are denoted in Table 3 . At day 0.5 the soil is still very wet and low RMSE values are observed. Larger differences in RMSE values between the coarse discretization and the a priori refined grids are already observed. In dryer soil regions the RMSE of scenario $2(0.5$ by 05 by $0.5 \mathrm{~cm})$ is about a factor of 6 higher than the corresponding a priori refined grid (scenario 5). Scenario 3 (1 by 1 by $1 \mathrm{~cm}$ ) and scenario 4 ( 2 by 2 by $2 \mathrm{~cm}$ ) differ with a factor larger than 10 and 20 , respectively, compared to their a priori refined grids. The coarse discretizations are not predicting the soil water potential distributions accurately. Maximum and mean errors in xylem water potential for the whole root structure are given in Table 4 for the three different simulation times. At day 0.5 (wet soil conditions) the coarse grid configurations have very large maximal errors $(>35 \%)$. The mean error though is still low (below 5\%), except for scenario 4. At day 4 and 5.4 (dryer soil conditions) the mean errors of the coarse discretizations become larger than 5\%. The a priori refined grids, conversely, show maximum errors at simulation time 5.4 of below $5.5 \%$ and mean errors near and below $2.5 \%$. The errors observed for the a priori refined grids (scenario 5-7), even in local dry soil conditions, can be considered marginal.

[21] Table 4 shows furthermore the total computational time $\left(T_{\mathrm{c}}\right)$ and number of soil nodes used for each simulation. Regular coarse grids are fast, even scenario 2 ( 0.5 by 0.5 by $0.5 \mathrm{~cm}$ ) needs only $14 \%$ of the time needed for the reference scenario. However, they give poor accuracy as observed before. On the other hand, a priori refined grids take approximately half the computational time of the reference grid (for this example), while keeping a very good accuracy for the water potential prediction as compared to the fine regular grid (reference). The lowest errors, from a soil and root point of view, are obtained with scenario 5 (one-level refinement scheme). Note that the number of soil nodes that were generated by the refinement approaches 5-7 differ, dependent on the initial soil spatial discretization and the level of refinement. No linear relation between the number of soil nodes and the soil spatial resolution, as was the case for regular grid configurations, can be found anymore. For this

Table 3. Root Mean Square Errors (cm) of the Soil Water Potentials in $x-y$ Cross Section at $z=-2 \mathrm{~cm}$ at Simulation Day $0.5,4$, and $5.4 \mathrm{~d}^{\mathrm{a}}$

\begin{tabular}{lccccccc}
\hline & \multicolumn{7}{c}{ Scenario } \\
\cline { 2 - 8 } & Reference $^{\mathrm{b}}$ & \multicolumn{1}{c}{2} & \multicolumn{1}{c}{3} & \multicolumn{1}{c}{4} & \multicolumn{1}{c}{5} & \multicolumn{1}{c}{6} \\
\hline RMSE at $t=0.5 \mathrm{~d}$ & - & 2.5 & 7.7 & 15.7 & 0.9 & 1.0 & 1.0 \\
RMSE at $t=4 \mathrm{~d}$ & - & 133.6 & 307.6 & 469.9 & 22.2 & 29.8 & 28.7 \\
RMSE at $t=5.4 \mathrm{~d}$ & - & 216.0 & 472.4 & 880.8 & 33.5 & 45.2 & 43.6 \\
\hline
\end{tabular}

${ }^{a}$ The errors are obtained by comparing the simulated scenarios of Table 1 with the reference scenario.

${ }^{\mathrm{b}}$ Minimum soil water potentials for the reference scenario at $t=0.5,4$, and $5.4 \mathrm{~d}$ are $-551,-9265$, and $-16614 \mathrm{~cm}$, respectively. 
Table 4. Xylem Water Potential $\left(\mathrm{WP}_{\mathrm{x}}\right)$ Errors for the Total Root Structure at Simulation Days 0.5, 4, and 5.4 and Computational Time $T_{\mathrm{c}}$ for the Simulated Scenarios of Table 1 Compared to the Reference Scenario ${ }^{a}$

\begin{tabular}{|c|c|c|c|c|c|c|c|}
\hline & \multicolumn{7}{|c|}{ Scenario } \\
\hline & Reference & 2 & 3 & 4 & 5 & 6 & 7 \\
\hline Max error in $\mathrm{WP}_{\mathrm{x}}(\%)$ at $t=0.5 \mathrm{~d}$ & - & 35.1 & 41.0 & 46.0 & 0.7 & 0.8 & 0.8 \\
\hline Mean error in $\mathrm{WP}_{\mathrm{x}}(\%)$ & - & 1.1 & 3.3 & 6.5 & 0.3 & 0.3 & 0.3 \\
\hline Max error in $\mathrm{WP}_{\mathrm{x}}(\%)$ at $t=4 \mathrm{~d}$ & - & 40.8 & 68.2 & 78.3 & 3.4 & 4.6 & 4.5 \\
\hline Mean error in $\mathrm{WP}_{\mathrm{x}}(\%)$ & - & 5.2 & 12.4 & 22.2 & 1.4 & 1.9 & 1.8 \\
\hline Max error in $\mathrm{WP}_{\mathrm{x}}(\%)$ at $t=5.4 \mathrm{~d}$ & - & 39.2 & 64.9 & 81.3 & 3.9 & 5.5 & 5.2 \\
\hline Mean error in $\mathrm{WP}_{\mathrm{x}}(\%)$ & - & 6.8 & 16.5 & 27.9 & 1.7 & 2.4 & 2.3 \\
\hline$T_{\mathrm{c}}$ compared to reference $(\%)$ & 100 & 14 & 6 & 4 & 47 & 46 & 49 \\
\hline Number of soil nodes & 230297 & 30429 & 4235 & 648 & 78312 & 69760 & 72113 \\
\hline
\end{tabular}

${ }^{\mathrm{a}}$ Furthermore, the number of soil nodes for each grid configuration is given.

root structure scenario 6 (two-level refinement) generates the fewest soil nodes. Moreover, the total computational time is the lowest.

\subsection{A Priori Versus A Posteriori Refinement}

[22] The static a priori grid from scenario 6 (two-level refinement, Table 1) is visually compared to two obtained grids using the adapted a posteriori error estimate in Figure 3. The adapted a posteriori error estimate creates a grid based on the uptake of water by all root segments. The left graph shows a transparent $\mathrm{XZ}$ view for the a priori grid, the middle graph of the a posteriori grid (post 1) with a $\theta_{\mathrm{E}}$ of 0.6 and $\theta_{\mathrm{o}}$ of 0.4 and the right graph of the a posteriori grid (post 4) with a $\theta_{\mathrm{E}}$ and $\theta_{\mathrm{o}}$ of respectively 0.5 and 0.2 . The a posteriori grids show that indeed soil voxels have a finer discretization near roots where larger water potential gradients are observed. In total, $99 \%$ of both a posteriori refined grids corroborate with the a priori refined grid. On
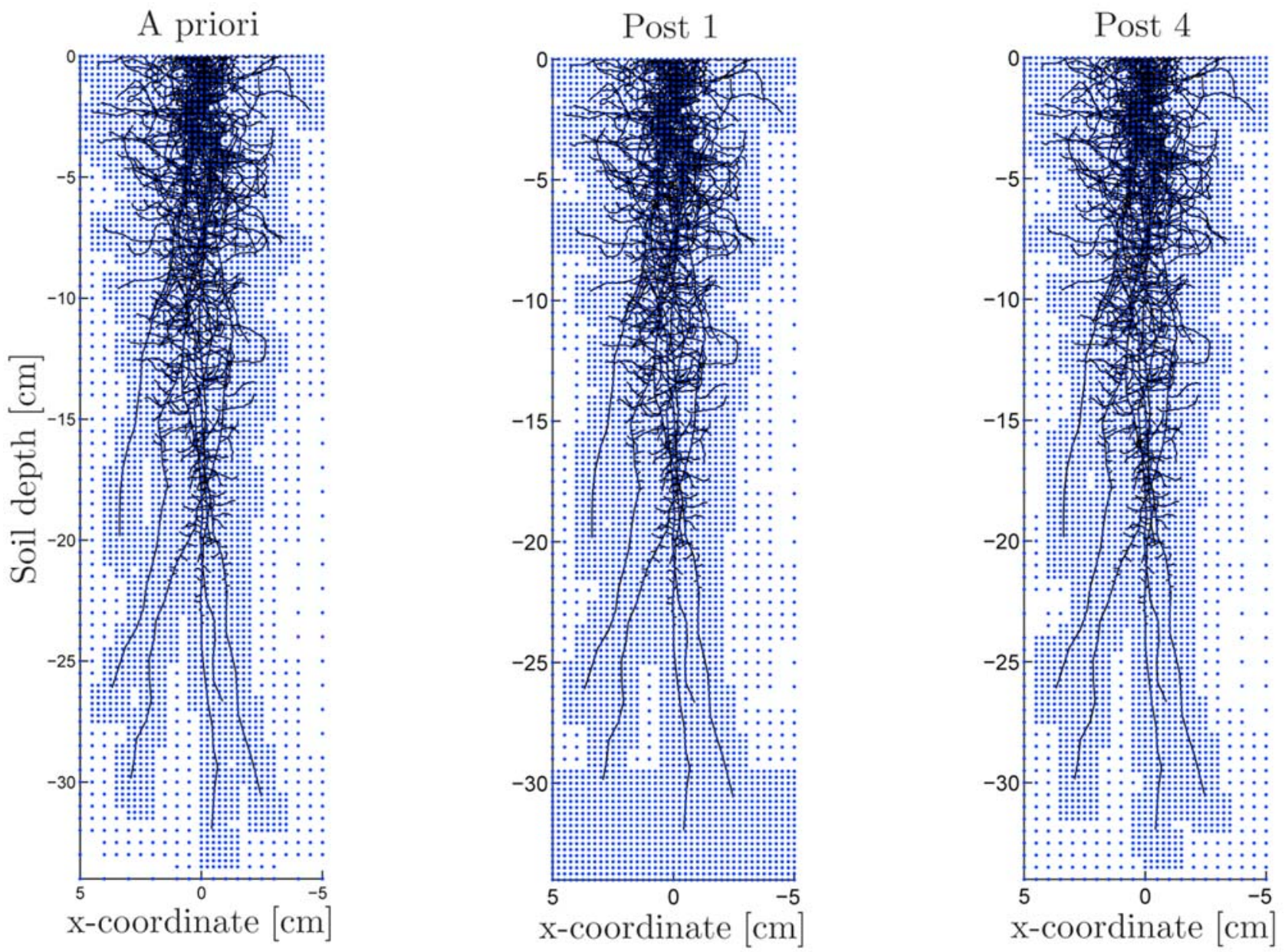

Figure 3. (left) $\mathrm{XZ}$ aspect of the a priori grid (two-level refinement; scenario 6). (middle) $\mathrm{XZ}$ aspect of the a posteriori grid with $\theta_{\mathrm{E}}=0.6, \theta_{\mathrm{o}}=0.4$ (scenario post 1 of Table 2). (right) XZ aspect of the a posteriori grid with $\theta_{\mathrm{E}}=0.5$ and $\theta_{\mathrm{o}}=0.2$ (scenario post 4 of Table 2). The solid lines denote root branches, whereas the dots represent soil nodes. 
Table 5. Xylem Water Potential $\left(\mathrm{WP}_{\mathrm{x}}\right)$ Errors for the Total Root Structure at Simulation Days 0.5, 4, and $5.4 \mathrm{~d}$ and Computational Time $T_{\mathrm{c}}$ for the Simulated A Posteriori Refinement Scenarios (Table 2) and Scenario 6 of Table 1 (A Priori Grid) compared to the Reference Scenario $^{\mathrm{a}}$

\begin{tabular}{|c|c|c|c|c|c|c|}
\hline & \multicolumn{6}{|c|}{ Scenario } \\
\hline & Reference & Pre-6 & Post-1 & Post-2 & Post-3 & Post-4 \\
\hline Max error in $\mathrm{WP}_{\mathrm{x}}(\%)$ at $t=0.5 \mathrm{~d}$ & - & 0.8 & 0.6 & 0.6 & 0.5 & 0.7 \\
\hline Mean error in $\mathrm{WP}_{\mathrm{x}}(\%)$ & - & 0.3 & 0.3 & 0.3 & 0.3 & 0.3 \\
\hline Max error in $\mathrm{WP}_{\mathrm{x}}(\%)$ at $t=4 \mathrm{~d}$ & - & 4.6 & 5.6 & 5.7 & 3.8 & 5.0 \\
\hline Mean error in $\mathrm{WP}_{\mathrm{x}}(\%)$ & - & 1.9 & 2.3 & 2.3 & 2.0 & 2.0 \\
\hline Max error in $\mathrm{WP}_{\mathrm{x}}(\%)$ at $t=5.4 \mathrm{~d}$ & - & 5.5 & 7.0 & 6.9 & 5.6 & 5.8 \\
\hline Mean error in $\mathrm{WP}_{\mathrm{x}}(\%)$ & - & 2.4 & 2.8 & 2.9 & 2.3 & 2.5 \\
\hline$T_{\mathrm{c}}$ compared to reference $(\%)$ & 100 & 46 & 260 & 210 & 280 & 170 \\
\hline Number of soil nodes & 230297 & 69760 & 106831 & 93220 & 100371 & 75151 \\
\hline
\end{tabular}

${ }^{\mathrm{a}}$ Furthermore, the number of soil nodes for each grid configuration is given.

the other hand, we notice for both scenarios differences in transition from coarser to finer soil cubes, other than a stepsize of one. Furthermore, the first depicted a posteriori grid (post $1, \theta_{\mathrm{E}}=0.6$ and $\theta_{\mathrm{o}}=0.4$ ) has more refined soil cubes in the lower soil regions compared to the second depicted a posteriori acquired grid. Note that there were no flux boundary conditions at the soil boundaries. The major cause of these differences is the sensitivity of the marking sequences. The higher the instituted value for $\theta_{\mathrm{E}}$ or $\theta_{\mathrm{o}}$ the more soil elements are selected for refinement. The results of scenario post 1 point out that after selection of those elements with a high error, other elements with lower errors were selected as well. These elements were located further away from the root structure, where no high water potential gradients and errors reside. For the three-dimensional soilroot water transfer model the choice of the marking sequence parameters is not straightforward. This can furthermore be observed from the number of soil nodes of the other acquired a posteriori grids in Table 5. Both the error and oscillation marking sequence parameters affect the number of refined cubes largely. The first a posteriori grid (post 1) creates a mesh with over 100.000 nodes, whereas the grid with a $\theta_{\mathrm{E}}$ of 0.5 and $\theta_{\mathrm{o}}$ of 0.2 (post 4 ) is much closer to the number of soil nodes of the a priori obtained grid. From all grids the a priori grid is with respect to the spatial distribution more optimal than the a posteriori grids. Not only because a minimum number of soil nodes is generated, but also because transitions in soil cubes from coarse to fine and vice versa differ only a stepsize of one.

[23] One of the adaptations for the usage of the a posteriori error estimate was that refinement was stopped after all soil cubes with a root segment, that takes up water, equal the minimum discretization size. This adaptation can be tested by analyzing the local error indicator in equation (A11). It was observed that the difference in local error indicator between the previous refined grid and the current refined grid started to converge to a small value. Furthermore, the maximum error was initially very large for the coarse soil discretization and was reduced for each time the grid was refined. When finally all soil cubes with root nodes in it equaled the minimum discretization size an acceptable maximum error, in comparison to the estimated soil water potential, was calculated. Moreover, there was no indication to interrupt the a posteriori refinement procedure earlier than the methodological imposed criterion that was implemented. This indicates that the criterion we imposed is not a bad assumption. Furthermore, the local error indicator was derived separately for the a priori obtained grid and showed that the maximum error was acceptable as well, in comparison to the estimated soil water potential. This indicates furthermore that a priori grids lead to a reduction in the error between exact and approximated solutions, compared to a coarse grid configuration, and that refinement was performed at correct locations where soil water potential gradients were expected to be larger, i.e., near roots. After the "static" grid is obtained with the adapted a posteriori error estimate we let the simulation run for a flux root collar boundary condition until stress is reached. A comparison of the xylem water potential error in Table 5 at the three simulation times $(0.5,4$ and $5.4 \mathrm{~d})$ shows that the errors obtained with the a posteriori refinement approach (compared to the reference scenario) are within range of the errors acquired with the a priori grid. Mostly they are slightly higher, however, for the post 3 case $\left(\theta_{\mathrm{E}}, \theta_{\mathrm{o}}=0.5\right)$ the xylem water potential errors are slightly lower. This is mainly caused by the large difference in number of soil nodes between those scenarios, secondly by the location where soil nodes are refined. The latter is dependent on $\theta_{\mathrm{E}}$ and $\theta_{\mathrm{o}}$ parameters. If in a soil layer (perpendicular to the axial direction) more fine soil cubes are generated the water potential gradient will be predicted steeper compared to the case where more coarse soil cubes are present, caused by the linear interpolation in elements [Schröder et al., 2008]. More important is the comparison of the computational time in Table 5. The computational time is significantly affected, it is even larger than the reference scenario for all a posteriori scenarios. This is mainly caused by the computational routines to estimate the local error for each element, furthermore because of additional routines to obtain a grid that ensures conservation requirements. For the a posteriori scenario $4\left(\theta_{\mathrm{E}}=0.5, \theta_{\mathrm{o}}=0.2\right)$ the time to generate the static grid with the adapted a posteriori error estimate is about 10 times longer than the time needed using the a priori approach.

\subsection{Dynamic A Priori Refinement Based on Active Root Segments}

[24] Simulations were performed for flux root collar boundary conditions. The errors in xylem water potential and the computational time of the dynamic a priori grid scenario, where refinement is based on the distribution of the active roots, are compared to the reference scenario in Table 6. Furthermore, the static a priori grid (scenario 6, 
Table 6. Xylem Water Potential $\left(\mathrm{WP}_{\mathrm{x}}\right)$ Errors for the Total Root Structure at Simulation Days 0.5, 4, and $5.4 \mathrm{~d}$ and Computational Time $T_{\mathrm{c}}$ for the Simulated Dynamic A Priori Refinement Scenario Compared to the Reference Scenario ${ }^{a}$

\begin{tabular}{lccc}
\hline & \multicolumn{3}{c}{ Scenario } \\
\cline { 2 - 4 } & Reference & $\begin{array}{c}\text { Static } \\
\text { A priori }\end{array}$ & $\begin{array}{c}\text { Dynamic } \\
\text { A priori }\end{array}$ \\
\hline $\begin{array}{l}\text { Max error in } \mathrm{WP}_{\mathrm{x}}(\%) \\
\text { at } t=0.5 \mathrm{~d}\end{array}$ & - & 0.8 & 4.3 \\
$\begin{array}{l}\text { Mean error in } \mathrm{WP}_{\mathrm{x}}(\%) \\
\text { Max error in } \mathrm{WP}_{\mathrm{x}}(\%)\end{array}$ & - & 0.3 & 0.7 \\
$\quad$ at $t=4 \mathrm{~d}$ & - & 4.6 & 10.3 \\
Mean error in $\mathrm{WP}_{\mathrm{x}}(\%)$ & - & 1.9 & 2.8 \\
Max error in $\mathrm{WP}_{\mathrm{x}}(\%)$ & - & 5.5 & 13.9 \\
$\quad$ at $t=5.4 \mathrm{~d}$ & - & 2.4 & 3.8 \\
Mean error in $\mathrm{WP}_{\mathrm{x}}(\%)$ & 100 & 46 & 37 \\
$T_{\mathrm{c}}$ compared to reference $(\%)$ & &
\end{tabular}

${ }^{\mathrm{a}}$ Furthermore, the static a priori scenario 6 (Table 1) is denoted for comparison purposes.

Table 1) is given to facilitate evaluation. Striking is the difference in maximum water potential error. Despite the fact that only those soil cubes are refined with a root segment that takes up water, the water potential gradient estimation from the outer soil column toward the roots is affected. It is the same principle as was mentioned in the previous section. In the dynamic approach more coarse cubes are available in a horizontal soil layer and the predicted water potential gradient from the soil outer boundary toward the root segments is calculated less steep, compared to the reference scenario. Although the maximum xylem water potential errors are rather large in the dynamic approach the mean errors are still below 5\% and acceptable. Furthermore, the errors are lower than the errors observed for the coarse grid configurations (Table 4). The dynamic approach based on a priori root information seems to be a suitable approach for simulating quickly and with high enough accuracy. The computational time is reduced by $20 \%$ compared to the static a priori grid. This is mainly caused by the reduction in number of soil nodes. Approximately 50\% less soil nodes were initially generated with the dynamic approach. Note that the grid was furthermore constant over time, as the observed differences in radial soil-root water flow (for the dynamic criterion) were less than $0.001 \%$, so that the criterion that was used to decide on grid refinement did not indicate a need to refine additionally. The dynamic approach can be applied on large soil and root structures that can be run on a single processor for a coarse soil discretization, but not for a regular fine soil discretization, or with the static approach. This is however dependent on the type of scenario and the available computer resources. Furthermore, root growth can easily be modeled with the dynamic refinement scheme.

\section{Conclusions}

[25] Because three-dimensional soil-root water transfer models cost lots of computational time for accurate prediction of water potential gradients in the soil and root system, different grid refinement techniques were evaluated. Because roots take up water and create large soil water potential gradients around them an a priori grid refinement technique was introduced based on the root architecture. It can either be used in a static or dynamic approach.

[26] Results from a soil-root scenario for two different root collar boundary conditions show that the accuracy of static a priori refined grids is maintained in comparison to a regular fine grid that serves as a reference. In contrast, regular coarse grids do not predict accurate solutions. Furthermore, due to the reduction of the grid complexity the computational time is reduced largely.

[27] The static grid that was obtained by a priori refinement can be obtained using a well recognized a posteriori refinement technique (adapted to obtain this static grid), indicating once more that the a priori obtained grid is well predicted at locations were roots reside. The disadvantages of the adapted a posteriori refinement technique, though, were firstly that the grids were spatially not as optimal as the a priori acquired grids; not a minimum number of soil nodes were generated, and not always a transition in soil cubes from coarse to fine and vice versa differed a stepsize of one. Secondly, no significant gain in accuracy, compared to the results using a priori grids, was obtained and thirdly the computational time to gain the static a priori grid was much larger than the a priori approach. As a consequence the total computational time for performed simulations exceeded the time of the reference scenario, which makes the usage of the adapted a posteriori refinement approach in these type of soil-root models questionable. On the other hand, if the point of departure is an a priori grid then the a posteriori refinement technique could be used whenever soil gradients, further away from the roots, get too large after time, e.g., in soil layers were the average water content is very low or due to rapidly changing soil boundary conditions [Mansell et al., 2002]. The latter, however, is implicitly considered in the a priori refinement technique. Using the a posteriori error estimator the grid could be extended such that a better accuracy could be acquired. Note that the trade off between computational costs of the a posteriori error estimator and the gain in accuracy/overall computational time should be minimized.

[28] Because only part of the root system is active a static a priori grid overestimates the required number of refined soil cubes. Therefore dynamic refinement is incorporated using the a priori refinement technique and shows that with an acceptable accuracy, compared to the reference case, the computational time can be reduced even more. The usage of dynamic refinement can be an advantage for modeling large soil and root structures with high accuracy for which, on single processors, only coarse soil and root discretizations with low accuracy can be run. Furthermore, dynamic methods can easily be used to model root growth, opposed to static refinement methods. In latter case it may occur that new grown roots are located in coarse soil cubes which may be undesirable if they start to take up water.

\section{Appendix A: A Posteriori Error Estimate for the Three-Dimensional Soil Water Flow Equation}

[29] The a posteriori error estimate is deduced for the Richards equation

$$
\frac{\partial \theta}{\partial t}=\nabla \cdot\left(K\left(\nabla \psi+\boldsymbol{e}_{z}\right)\right)-S_{t}
$$


where $\theta$ is the volumetric soil water content $\left[\mathrm{cm}^{3} \mathrm{~cm}^{-3}\right]$, $K(\psi)\left[\mathrm{cm} \mathrm{d}^{-1}\right]$ the soil hydraulic conductivity, $S_{t}\left[\mathrm{~d}^{-1}\right]$ the sink term regulating root water uptake, $t[\mathrm{~d}]$ the time and $\boldsymbol{e}_{z}=\nabla z$ the unit vector field in the vertical direction. The time derivative of the water content is related to the water potential by $\frac{\partial \theta}{\partial t}=C(\psi) \frac{\partial \psi}{\partial t}$, where $C(\psi)\left[\mathrm{cm}^{-1}\right]$ is the soil capacity. We use an implicit backward Euler scheme that transforms equation (A1) in

$$
\frac{\theta_{n+1}-\theta_{n}}{\triangle t}=\nabla \cdot\left(K\left(\nabla \psi_{n+1}+\boldsymbol{e}_{z}\right)\right)-S_{t}
$$

where the subscript ${ }_{n}$ denotes the previous time step, the subscript $_{n+1}$ the current time step and $\nabla t$ the time stepsize. Equation (A2) can be rewritten as

$$
-\nabla \cdot\left(K \nabla \psi_{n+1}\right)+\frac{\theta_{n+1}}{\triangle t}=f_{n}
$$

where $f_{n}=\nabla \cdot\left(K \boldsymbol{e}_{z}\right)+{ }^{\theta_{n}} / \triangle t-S_{t}$.

[30] To find the solution $\psi \in \mathbb{V}$ for each time step we need the weak form of equation (A3) over the domain $\Omega$ such that

$$
\begin{aligned}
\mathcal{B}[\psi, v] & =\int_{\Omega}\left(K \nabla \psi \cdot \nabla v+\frac{\theta}{\triangle t} v\right) d \Omega+\int_{\Gamma}(K \nabla \psi v) \cdot \boldsymbol{n} d \Gamma \\
& =\int_{\Omega} f v d \Omega=\mathcal{F}(v) \quad \forall v \in \mathbb{V}
\end{aligned}
$$

where $v$ is a weight function and $\boldsymbol{n}$ the outward unit vector normal to boundary $\Gamma$. Note that we dropped the time index. The discrete weak form is then given by

$$
\mathcal{B}\left[\psi_{H}, v\right]=\mathcal{F}(v) \quad \forall v \in \mathbb{V}_{H}
$$

where $\psi_{H} \in \mathbb{V}_{H}$ is the approximated solution.

[31] The weight functions are derived for linear tetrahedral elements [Cheng and Zhang, 2007]. Assembling of the system of equations is performed as was done by Simunek et al. [1995]. After the linear system of equations is solved with some method we can evaluate the error that emerges when approximating the solution. Substitution of the error $e_{H}:=\psi-\psi_{H}$ (difference between exact and approximated solution) into the bilinear form $\mathcal{B}\left[e_{H}, v\right]$ (equation (A5)) and integrate by parts elementwise the so-called error representation formula is obtained [Nochetto, 2006]

$$
\mathcal{B}\left[e_{H}, v\right]=\sum_{T \in \mathcal{T}_{H}} \int_{T} R_{T}\left(\psi_{H}\right) v+\sum_{S \in \mathcal{S}_{H}} \int_{S} J_{S}\left(\psi_{H}\right) v \quad \forall v \in \mathbb{V}
$$

The left part $R_{T}\left(\psi_{H}\right)$ is associated with the element residual, the right part $J_{S}\left(\psi_{H}\right)$ with the jump residual; similarly to the left handside of equation (A4).

[32] For a tetrahedral element $\mathrm{T}$ of the mesh $\mathcal{T}_{H}$ the element residual is defined as

$$
R_{T}\left(\psi_{H}\right)=f+\nabla \cdot\left(K \nabla \psi_{H}\right)-\frac{\theta_{H}}{\triangle t}
$$

where $\theta_{H}$ is the water content that belongs to the approximated solution variable $\psi_{H}$. For the set of interior faces $\mathcal{S}_{H}$ of the mesh $\mathcal{T}_{H}$ the jump residual is defined as

$$
J_{S}\left(\psi_{H}\right)=-K \nabla \psi_{H}^{+} \cdot \nu^{+}-K \nabla \psi_{H}^{-} \cdot \nu^{-}
$$

where $S$ is the common side of elements $T^{+}$and $T^{-}$with unit outward normals $\nu^{+}$and $\nu^{-}$, respectively. From this we can deduce the local error indicator $\eta_{H}(T)$ by

$$
\eta_{H}(T)^{2}:=H_{T}^{3}\left\|R_{T}\left(\psi_{H}\right)\right\|_{L^{2}(T)}^{2}+\sum_{S \subset \partial T} H_{S}^{2}\left\|J_{S}\left(\psi_{H}\right)\right\|_{L^{2}(S)}^{2}
$$

where $H$ stands for the mesh size and $L^{2}$ is the vector space. The element residual is analog to the residual in linear algebra and describes the relation between the error $e_{H}$ and residual for the vertices of an element. The jump residual evaluates the boundaries of the elements.

[33] Another important concept, oscillation, accounts for information missed by the averaging process associated with the finite element method. The oscillation on the elements $T \in \mathcal{T}_{H}$ is defined by

$$
\operatorname{osc}_{H}(T)^{2}:=H_{T}^{3}\left\|R_{T}-\overline{R_{T}}\right\|_{L^{2}(T)}^{2}
$$

where $\overline{R_{T}}$ is denoted by $\frac{\int_{T} R_{T} d T}{v^{2} l_{T}}$, where $\operatorname{vol}_{T}$ is the volume of the element.

[34] Important inequalities that belong to equations (A9) and (A10) can be deduced [Nochetto, 2006]. There exist constants $C_{1}, C_{2}>0$ such that

$$
\begin{aligned}
& \left\|\left|\psi-\psi_{H}\right|\right\|^{2} \leq C_{1} \eta_{H}(\Omega)^{2} \\
& C_{2} \eta_{H}(T)^{2} \leq\left\|\psi-\psi_{H}\right\|_{H^{1}\left(w_{T}\right)}^{2}+\operatorname{osc}_{H}\left(w_{T}\right)^{2}
\end{aligned}
$$

where the energy norm is given by $\|v \mid\|=\mathcal{B}[v, v]^{1 / 2}$, $\Omega$ consists of all elements and $w_{T}$ of all elements sharing at least one face with $T$. The first inequality tells us that the sum of the element residual and jump residual is a reliable upper bound, the latter inequality indicates that $\eta_{H}(T)$ is a locally sharp error estimate provided that the oscillation is small. This leads to two obvious marking strategies for a subset of elements $\hat{T}_{H}$ of the mesh $\mathcal{T}_{H}$ such that after refining, both error and oscillation are reduced. These strategies are given in equations (4) and (5).

[35] After the soil system is solved the local error indicator (equation (A9)) is calculated and evaluation of the upper inequality in equation (A11) indicates if refinement is needed. If so, two marking procedures can be chosen dependent on the strategy of refinement.

\section{Appendix B: Addendum}

[36] This addendum is developed to assist in implementing the a priori refinement theory as explained in the main text. The first assumption is that the spatial coordinates of the grid, representing in this case the soil system, and of the root structure are known. In addition, the element information containing node id's is known, i.e., which nodal coordinate belongs to an element. The soil grid is assumed to be divided into cubes and each cube consists of six 


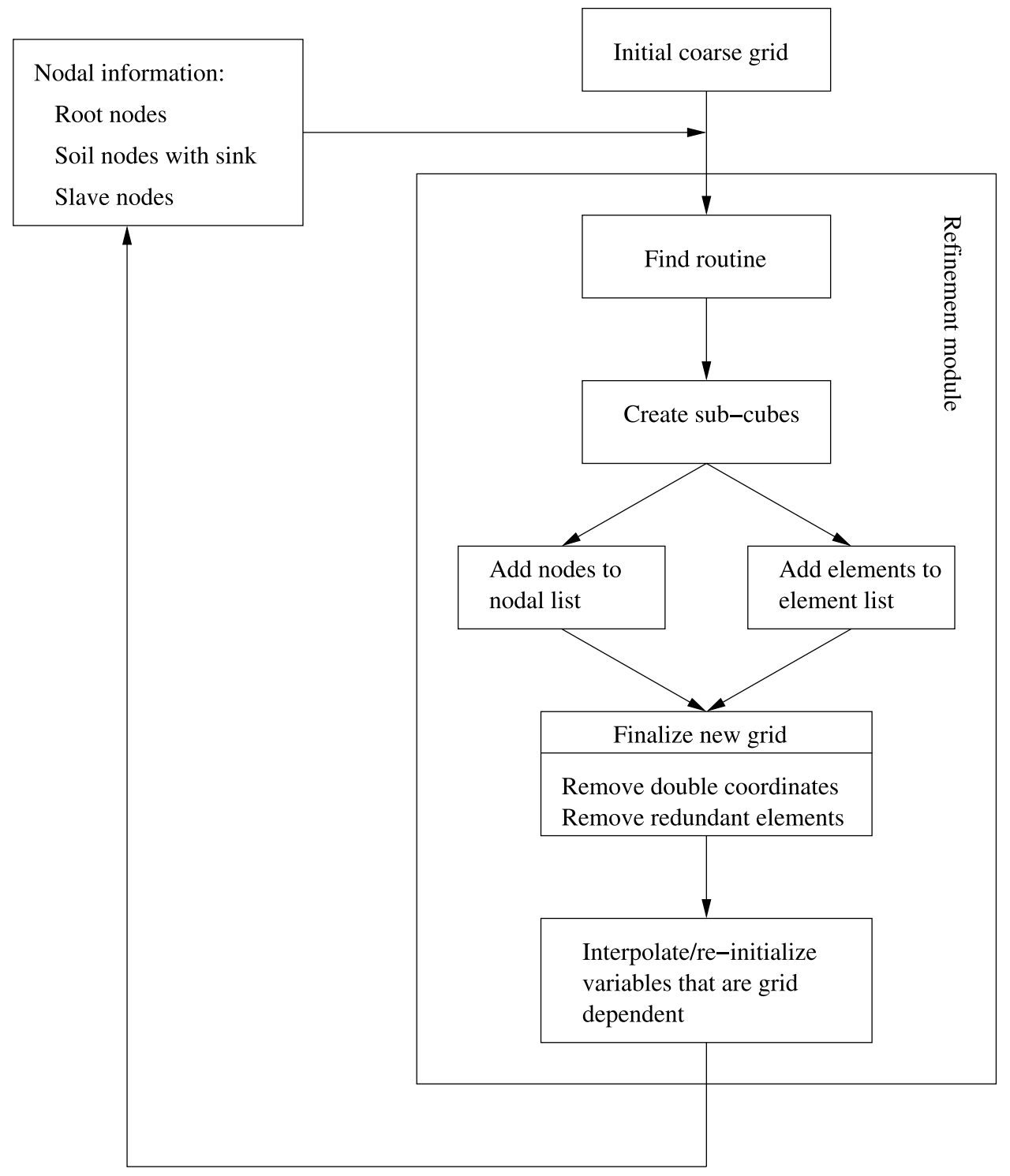

Figure B1. Flowchart of the refinement module.

tetrahedral elements of equal size. An overview of the refinement module is given in Figure B1.

[37] The first goal is to find the coordinates of the eight cube vertices that surround a root node. This identified soil cube should be subdivided into smaller cubes.

\section{B1. Finding a Root Node in a Cube}

[38] This is a short algorithm that can be obtained using several methods. Two methods are explained hereafter.

[39] Needed: increment length of a soil cube in $\mathrm{x}, \mathrm{y}$ and $\mathrm{z}$ direction $(d x, d y, d z)$

[40] Alternative: minimum $\mathrm{x}$ and $\mathrm{y}$ coordinate of the soil grid $(\min X, \min Y)$; maximum $\mathrm{z}$ coordinate of the soil grid (maxZ). Number of elements in $\mathrm{x}$ and $\mathrm{y}$ direction (nex, ney)

[41] 1. A simple algorithm, but costs more computational time

[42] - consider a root node $\left(x_{\text {root }}\right)$

[43] - make a loop over all $\mathrm{x}$ coordinates of the soil nodes $\left(x_{\text {soil }}\right)$, for each nodal coordinate check whether the $\mathrm{x}$ coordinate lies in the range $x_{\text {root }}-d x \leq x_{\text {soil }} \leq x_{\text {root }}+d x$ do the same, for the $\mathrm{y}, \mathrm{z}$ coordinates, but with a loop over the previously nodes that were found to be in range

[44] - finally eight soil nodes are found to be used for further processing

[45] 2. An algorithm that uses less computational resources uses the properties of the regular grid and the consecutive numbering of elements with its coordinates

$$
\begin{gathered}
\delta_{1}=x_{\text {root }}-(\min X)-1 e-6 \\
i_{x_{\min }}=1+\text { floor }\left(\delta_{1} / d x\right) * 2 \\
\delta_{1}=y_{\text {root }}-(\min Y)-1 e-6 \\
i_{x y_{\min }}=i_{x_{\text {min }}}+\text { floor }\left(\delta_{1} / d y\right) * \text { nex } \\
\delta_{1}=\operatorname{abs}(\operatorname{root}(3)-\max Z)+1 e-6 \\
i_{\min }=i_{x y_{\min }}+\text { floor }\left(\delta_{1} / d z\right)^{*} \text { nex*ney }
\end{gathered}
$$



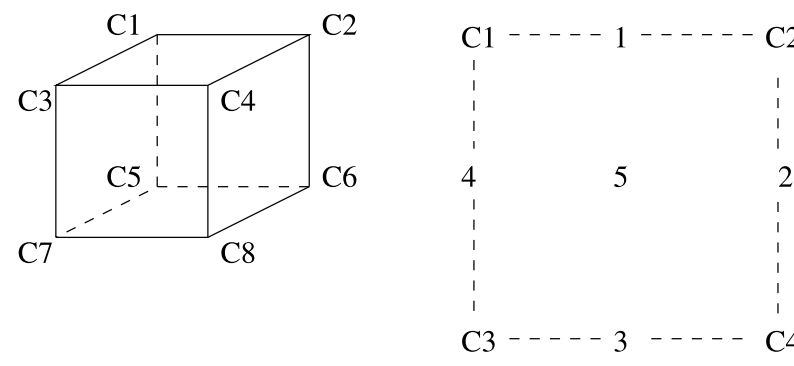

\section{Top plane} C4
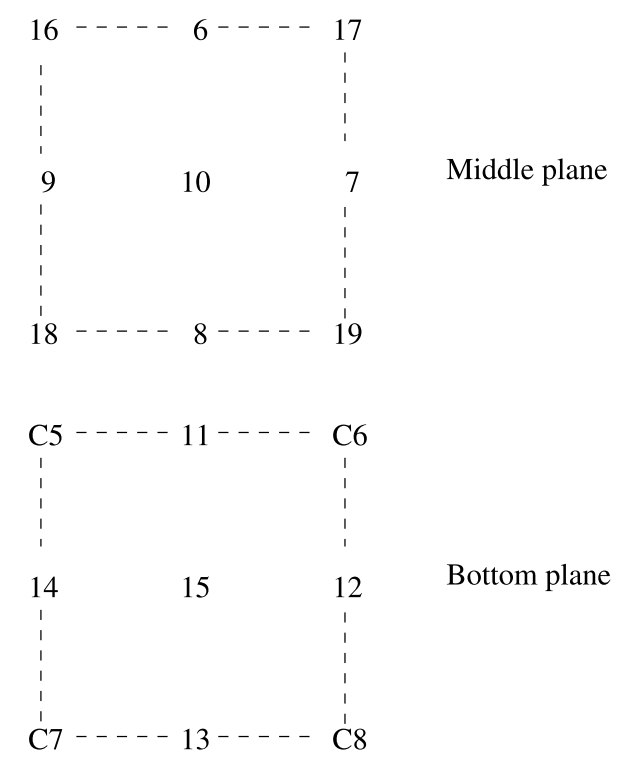

Figure B2. Subdividing a soil cube into eight additional cubes: generating additional 19 nodes for the subcubes. The corner nodes of the coarser cube are denoted by $\mathrm{C} 1-\mathrm{C} 8$.

The variable $i_{\min }$ gives the cube number that consists of the vertices surrounding the root node. Note that this algorithm is very specific to the study at hand.

\section{B2. Creating Subcubes}

[46] If the eight corner nodes of the soil cube surrounding the root node are identified the cube can be subdvided into smaller cubes (up to a level of refinement that is desired). Nineteen new coordinates are created from the eight already existing nodes, see Figure B2. These generated coordinates are added to the already existing coordinate list. Furthermore, it is checked whether the newly generated coordinates were already created. If so, their index number in the coordinate list is marked, such that after the process of refinement, these redundant coordinates can be removed.

[47] Besides the generation of new coordinates the element list should be updated as well. The eight subcubes have six new elements each that need to be listed (placed at end of element list). The elements that initially exist (for the coarser soil cube) need to be removed. The problem is that from the coordinate list, double entries were removed. The element list contains coordinate id's. If a node was removed, the coordinate next in the list has a lower number then actually listed in the element information. Therefore the coordinate values in the element list have to be adjusted.
This is done by a loop over all removed soil coordinates, that are checked with each coordinate entry in the element list. If the coordinate value in the element list is larger than the one removed, then reduce that guy by one; update the removal coordinate list as well.

[48] The only possible way to remove coordinate and element entries is to mark each refined cube. With this index information one can track each coordinate and element entry in their lists, respectively.

[49] The above listed procedure initiates refinement only for one level. If the level of refinement is larger than one, additional refinement of the just refined soil cube is desired. The root node is now searched in the newly generated eight cubes, similarly as in the previous section. The same routines are applied (but the search in which soil cube the root node lies costs less effort: only eight cubes have to be searched). The tracking of the new generated soil cubes is more difficult, eight new cubes are needed, with tracking numbers. This is far more extensive for a refinement level larger than two. Until now the refinement routine is capable to save the indices of three level of refinements. An overview of the tracking system is given in Figure B3.

[50] If a soil cube is already refined, then the tracking number is retrieved to find the created coordinates and 


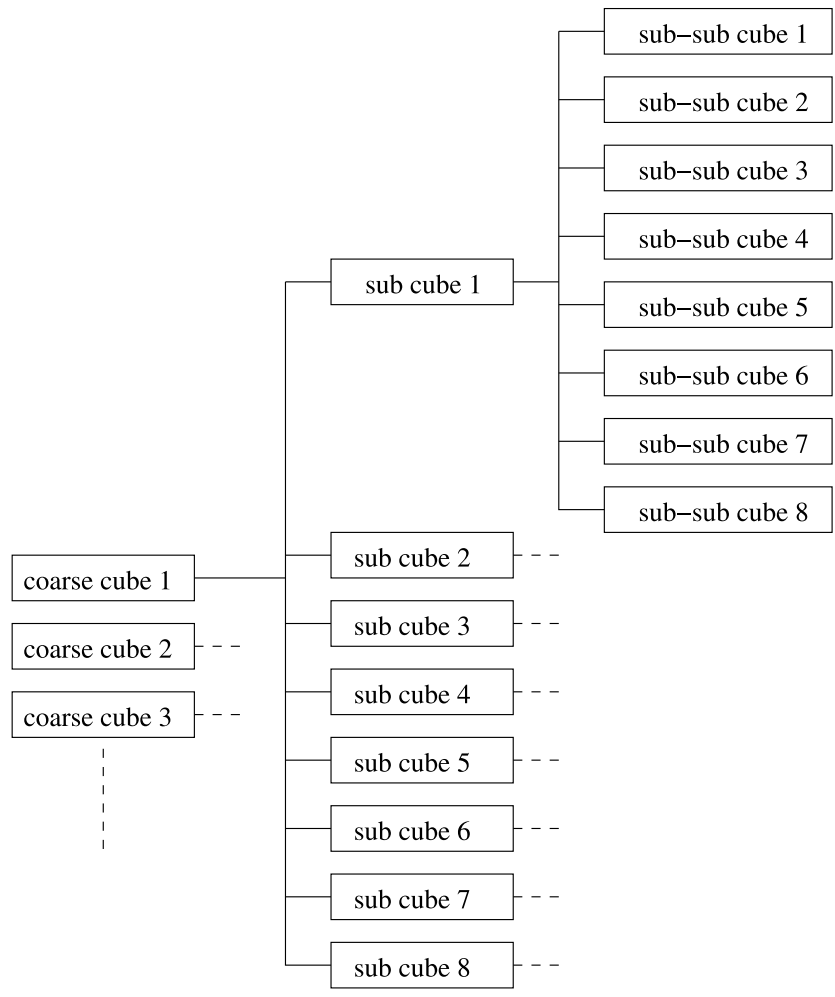

Figure B3. Tracking scheme of cubes. Start first to look for a coarse cube. If refined, look within the eight subcubes, etc.

element info in the coordinate and element list, respectively. Refinement is considered from then on. If this soil cube was already refined though, then no additional refinement is needed.

\section{B3. Extension of the Grid}

[51] The general refinement method described above can be used for multiple goals. For example, if we consider the soil-root problem as discussed in this study, we obtain, after refinement of all the soil cubes with a root node in it, refined cubes that possess vertices with a denoted sink term. If such a vertex is a slave node and has a sink term value larger than zero the grid around the slave node should be extended.

[52] Slave nodes: To generate a list with slave nodes one need to know how many neighbors it has. This is counted, furthermore, based on the geometry of the node it is calculated how many entries there should be, from this one can deduce if a soil node is a slave node or a master node.

[53] One could make a loop over all slave nodes, check whether they have a sink value larger than zero, if so, then take the cubes in which this slave node lies and refine those cubes using the refinement routine.

[54] A different application of refinement is to check whether the soil cube next to a slave node differs a stepsize of one in the level of refinement, if not, then this cube should be refined, one level lower than its original value (e.g., if the cube with the slave node has a cube length of $0.25 \mathrm{~cm}$ and the cube next to slave node has a length of 1 $\mathrm{cm}$, then the cube next to the slave node should be refined to a length of $0.5 \mathrm{~cm}$ ).
[55] Top and bottom boundary conditions can be refined by using the refinement routine as well. If top boundary conditions are imposed (e.g., rainfall) then the cubes in the topsoil layer are refined to the lowest instituted refinement level (e.g., $0.25 \mathrm{~cm})$ and the cubes underneath it are one level larger until the coarse cube configuration is reached.

[56] After each change in grid configuration, the final coordinate and element lists have to be compiled. Thus the lists where the newly generated coordinates and elements are added are finalized by removing redundant coordinates and elements.

\section{B4. Operations That Need to be Performed After the New Grid Is Obtained}

[57] This is dependent of the problem under investigation. Normally, the same routines that deal with the grid configuration have to be reinitialized (also boundary conditions). For example, the material parameter list. All nodal coordinates possess a value representing the soil material they belong too. If the soil exists of one material only, then there exists an array with a length equal to the number of nodal coordinates with each entry value equal to one. This array should be reinitialized, as some entry values, due to the new generated nodal coordinate list, are declared empty.

[58] Variables that are also dependent of the grid configuration are of course the variables within the Richards equation (water potential, water content, sink term). Interpolation of these variables is required. This can be obtained directly after the new coordinate and element lists are finalized. A new generated soil node is considered, and the soil nodes around this guy are retrieved. Because the location of the surrounding nodes may vary, due to different generated cube sizes, a loop over all possible dimensions of the soil cube must be made (e.g., starting with a coarse grid of 1 by 1 by $1 \mathrm{~cm}$, two-level refinement; then first the surrounding perimeter with a radius of $0.25 \mathrm{~cm}$ is checked, if no connecting nodes are found then a distance of $0.5 \mathrm{~cm}$ is used to find neighboring nodes). First in the horizontal $(\mathrm{x}, \mathrm{y})$ direction all adjacent nodes of the newly generated soil node are identified and interpolation is performed, if no interpolation is possible anymore, vertical interpolation is performed for those soil nodes that were formed in another soil layer but had no neighboring nodes in the previous coarser soil grid. Horizontal and vertical direction is alternated, until all new nodes have interpolated values.

\section{References}

Babuška, I., and W. C. Rheinboldt (1978), A posteriori error estimates for the finite element method, Int. J. Numer. Methods Eng., 12, 1597-1615. Bear, J. (1972), Dynamics of Fluids in Porous Media, Elsevier, New York. Briggs, W. L., V. E. Henson, and S. F. McCormick (2000), A Multigrid Tutorial, 2nd ed., SIAM, Philadelphia, Pa.

Carsel, R. F., and R. S. Parrish (1988), Developing joint probability distributions of soil water retention characteristics, Water Resour. Res., 24(5), 755-769.

Cheng, Y. M., and Y. H. Zhang (2007), Formulation of a three-dimensional numerical manifold method with tetrahedron and hexahedron elements, Rock Mech. Rock Eng., doi:10.1007/s00603-006-0120-9.

Doussan, C., L. Pagès, and G. Vercambre (1998), Modelling of the hydraulic architecture of root systems: An integrated approach to water absorption-distribution of axial and radial conductances in maize, Ann. Bot., 81, 225-232.

Javaux, M., T. Schröder, J. Vanderborght, and H. Vereecken (2008), Potential use of detailed modeling approach for predicting root water uptake, Vadose Zone J., 7, 1079-1088. 
Li, Y., M. Fuchs, S. Cohen, Y. Cohen, and R. Wallach (2002), Water uptake profile response of corn to soil moisture depletion, Plant Cell Environ., 25, 491- 500 .

Mansell, R. S., L. Ma, L. R. Ahuja, and S. A. Bloom (2002), Adaptive grid refinement in numerical models for water flow and chemical transport in soil: A review, Vadose Zone J., 1, 222-238.

Nochetto, R. H. (2006), Adaptive Finite Element Methods for Elliptic PDE CNA Summer School, Probabilistic and Analytical Perpectives in Contemporary PDE.

Pagès, L., G. Vercambre, J. Drouet, F. Lecompte, C. Collet, and J. Le Bot (2004), Root Typ: A generic model to depict and analyse the root system architecture, Plant Soil, 258, 103-119.

Passioura, J. B. (1980), The transport of water from soil to shoot in wheat seedlings, J. Exp. Bot., 31, 333-345.

Pohlmeier, A., A. Oros-Peusquens, M. Javaux, M. I. Menzel, J. Vanderborght, J. Kaffanke, S. Romanzetti, J. Lindenmair, H. Vereecken, and N. J. Shah (2008), Changes in soil water content resulting from ricinus root uptake monitored by magnetic resonance imaging, Vadose Zone J., 7, 1010-1017.

Schröder, T., M. Javaux, J. Vanderborght, B. Körfgen, and H. Vereecken (2008), Effect of local soil hydraulic conductivity drop using a 3D root water uptake model, Vadose Zone J., 7, 1089-1098.

Schröder, T., M. Javaux, J. Vanderborght, B. Körfgen, and H. Vereecken (2009), Implementation of a microscopic soil-root hydraulic conductivity drop function in a 3D soil-root architecture water transfer model, Vadose Zone J., 8(3), 783-792.
Šimunek, J., K. Huang, and M. Th. van Genuchten (1995), The SWMS 3D code for simulating water flow and solute transport in three-dimensional variably saturated media, V. 1.0 Research Rep. No. 139, U.S. Salinity Lab., ARS USDA, Riverside, Calif.

Somma, F., J. W. Hopmans, and V. Clausnitzer (1998), Transient threedimensional modeling of soil water and solute transport with simultaneous root growth, root water and nutrient uptake, Plant Soil, 202(2), $281-293$.

van Genuchten, M. Th. (1980), A closed-form equation for predicting the hydraulic conductivity of unsaturated soils, Soil Sci. Soc. Am. J., 44, $892-898$.

Wilderotter, O. (2003), An adaptive numerical method for the Richards equation with root growth, Plant Soil, 251, 255-267.

M. Javaux, J. Vanderborght, and H. Vereecken, Institute for Chemistry and Dynamics of the Geosphere, Agrosphere Institute, ICG-4, Forschungszentrum Jülich GmbH, D-52425 Jülich, Germany.

B. Körfgen and T. Schröder, Jülich Supercomputing Center, Forschungszentrum Jülich GmbH, D-52425 Jülich, Germany. (t.g.m.schroder@ gmail.com)

L. Tang, Department of Applied Mathematics, University of Colorado, 526 UCB, Boulder, CO 80309-0526, USA. 